\title{
Quercetin induces apoptosis and necroptosis in MCF-7 breast cancer cells
}

\author{
Khorsandi $\mathrm{L}^{1}$, Orazizadeh $\mathrm{M}^{2}$, Niazvand $\mathrm{F}^{1}$, Abbaspour $\mathrm{MR}^{2}$, Mansouri $\mathrm{E}^{1}$, Khodadadi $\mathrm{A}^{3}$ \\ Cell \& Molecular Research Center, Faculty of Medicine, Ahvaz Jundishapur University of Medical Sciences, \\ Ahvaz, Iran.niazvandf@gmail.com
}

\section{ABSTRACT}

OBJECTIVE: This study investigated the quercetin (Que) effects on growth of MCF-7 human cancer breast cell line and its cellular death mechanism.

BACKGROUND: Quercetin has been found to be very efficacious against many different types of cancer cells. However, the study is not sufficiently powered to demonastrate anticancer mechanisms.

METHODS: MCF-7cells were treated by $50 \mu \mathrm{M} / \mathrm{ml}$ of Que for 48 hours. MCF-7 cells were also pretreated with $10 \mathrm{Mm}$ ZVAD (apoptosis inhibitor) or $3 \mathrm{mM} \mathrm{Nec}-1$ (necroptosis inhibitor) for evaluation of cell death induced by apoptosis or necroptosis.

RESULTS: MTT and clonogenicity assays revealed that the Que induced a significant increase in cell viability and proliferation in presence of Nec-1 in comparison to the presence of ZVAD $(p<0.05)$. Que also increased apoptosis as revealed by DAPI staining and morphology evaluations. Following Que treatment Bcl-2 expression was significantly decreased while Bax expression was significantly increased. Que in presence of Nec-1 decreased expression of Bax gene, reduced apoptotic index, increased cell viability and proliferation of MCF-7 cells in comparison to absence of Nec-1. MCF-7 cells showed a significantly increased expression of RIPK1 and RIPK3 in response to Que plus ZVAD in comparison to absence of ZVAD.

CONCLUSION: Our results revealed that the high Que toxicity for breast cancer cells depends on multiple cell death pathways, which involve mainly necroptosis (Fig. 6, Ref. 21). Text in PDF www.elis.sk.

KEY WORDS: necroptosis, apoptosis, quercetin, breast cancer.

\section{Introduction}

Breast cancer is the most frequently diagnosed cancer and the leading cause of cancer deaths among females worldwide. The best lines of defense, radiation therapy and chemotherapy, are unsatisfactory due to the untoward side effects on healthy cells and the problem of drug resistance (1). Searching for new compounds for the treatment of cancer is the aim of numerous studies, and many works are focused on plant-derived compounds that have curative potential. Quercetin (Que) is one of the most abundant naturally occurring flavonoid and it has been found to be very efficacious against many kinds of cancer cells $(2,3)$.

${ }^{1}$ Cell \& Molecular Research Center, Faculty of Medicine, Ahvaz Jundishapur University of Medical Sciences, Ahvaz, Iran, ${ }^{2}$ Targeted Drug Delivery Research Center, School of Pharmacy, Mashhad University of Medical Sciences, Mashhad, Iran, and ${ }^{3}$ Department of Immunology and Cancer, Petroleum Pollutants Research Center, Ahvaz Jundishapur University of Medical Sciences, Ahvaz, Iran

Address for correspondence: F. Niazvand, Cell \& Molecular Research Center, Faculty of Medicine, Ahvaz Jundishapur University of Medical Sciences, P. O. Box 61335, Ahvaz, Iran.

Phone: +98.611.3720458, Fax: +98.611.3336380

Acknowledgement: This paper is a part of Ph.D. thesis of Firoozeh Niazvand and was supported by a Grant (CMRC-9418) from the research council of Ahvaz Jundishapur University of Medical Sciences.
Defects in cancer cell death are the most frequent causes of therapeutic resistance, and thus exploring cancer cell death might inform development of strategies to overcome therapeutic resistance (4). Apoptosis is type-I Programmed cell death (PCD) that is morphologically characterized by cell shrinkage, chromatin condensation, nuclear fragmentation and formation of apoptotic bodies (5). Apoptosis is regulated in part by Bcl-2 genes, which promote cell survival and pro-apoptotic protein Bax. Apoptosis inhibition depends partly on the balance between Bcl-2 and Bax expression (6). Necrosis has for a long time been considered an accidental mode of cell death. However, it was recently reported that a form of cell death morphologically classified as necrosis (termed necroptosis) could also be regulated in a programmed manner via defined signal transduction pathways (4). Tumor necrosis factor (TNF) receptor 1 triggers a signaling reaction that culminates in binding of receptor interacting protein kinase 3 (RIPK3) with its upstream activator RIPK1. RIPK1 and RIPK3 phosphorylation stabilizes this complex and promotes its conversion to an amyloid-like filamentous structure termed the necrosome (7). The necroptosis machinery is often impaired during tumorigenesis and tumor progression (8).

The idea that Que elicits more than one cell death pathway and the identification of mechanisms involved in cell killing would provide useful insights for the potential application of Que in cancer therapy. 


\section{Materials and methods}

\section{Experimental design}

The human MCF-7 and MCF-10a cell lines were obtained from Pasteur Institute of Iran. Control group was untreated cells. Experimental groups were treated by $50 \mu \mathrm{M} / \mathrm{ml}$ of Que. The dose of Que was selected based on our pilot study. Briefly, before the experiment, we examined different doses of Que $(0.5,1,5,25$, 50,75 and $100 \mu \mathrm{M} / \mathrm{ml}$ ) for 48 hours to determine the best one for anticancer action and 3 flasks were used for each dose. By using MTT assay the percentage of viable cells was significantly decreased at the concentrations more than $50 \mu \mathrm{M} / \mathrm{ml}$ (results not shown). There was no significant difference between concentration of 50 and $75 \mu \mathrm{M} / \mathrm{ml}$. Thus, we used Que at the dose of $50 \mu \mathrm{M} /$ $\mathrm{ml}$ in this experiment. MCF-7 cells were pretreated with $10 \mathrm{Mm}$ ZVAD (apoptosis inhibitor) or $3 \mathrm{mM} \mathrm{Nec}-1$ (necroptosis inhibitor) for 1 hour before treatment with Que for evaluation of cell death induced by apoptosis or necroptosis.

\section{Cell viability}

MTT (3-(4, 5-Dimethylthiazol-2-yl)-2, 5-diphenyltetrazolium-bromide) assay was used to evaluate the effect of Que on cell viability. Briefly, MCF-7 and MCF-10a cells were maintained with culture media for 48 hours in 24 well plates. MTT $(0.5 \mathrm{mg} /$ $\mathrm{ml}$ ) solution was then added to each well, and cells were further incubated for 4 hours at $37^{\circ} \mathrm{C}$. Supernatants were then removed, and $700 \mu \mathrm{l}$ of DMSO were added to each well to dissolve the formazan product. Absorbance at $540 \mathrm{~nm}$ was measured using a microplate reader (BioRad, CA). MTT assay values for labeled cells were expressed as the percentage of corresponding average values in control cells (9).

\section{Clonogenicity assay}

The anti-proliferative effect of Que on MCF-7 cells was measured by a colony formation assay. Five hundred cells were seeded into 6-well culture dishes. The next day, cells were exposed to Que in serum-free medium at the appropriate times. Cells were washed and further incubated with complete medium until further analysis. After incubation for 10 days, the cells were washed with $1 \times$ PBS and fixed with $4 \%$ paraformaldehyde at room temperature for 20-30 min. After washing with 1× PBS again, the cells were stained with $0.1 \%$ crystal violet/PBS, and the colonies were counted. All experiments were carried out in triplicate. The colonies which had $>50$ cells per colony were counted. Data were represented as number of colonies formed per 500 cells plated and converted to percentage (10).

\section{DAPI staining}

Five hundred cells were seeded into 6-well culture dishes. The MCF-7 and MCF-10a cells were grown on cover slips after treatment with Que for 48 hours. Nuclei changes of apoptosis were assayed by DAPI staining as previously described. Apoptotic index was calculated by dividing the number of apoptotic cells in a randomly selected microscopic field by the total number of cells in that field, and the result was multiplied by 100 . The Apoptotic indexes of 10 random fields were evaluated and the means of each case were calculated (11).

\section{Real time polymerase chain reaction}

Using the RNeasy Mini kit (Qiagen), RNA was isolated from the harvest cells according to manufacturer's instructions. cDNA was produced from the extracted RNAs using the cDNA synthesis kit based on the manufacturer's instructions (Fermentas, Canada). Approximately $2 \mu \mathrm{l}$ of cDNA was amplified in each $25 \mu \mathrm{l}$ PCR reaction mix containing $12.5 \mu \mathrm{l}$ of $2 \mathrm{x}$ SYBR Green Master Mix (Fermentas, Canada), $0.2 \mu \mathrm{l}$ of each 10 pmol forward and reverse primers and 10.1 $\mu \mathrm{l}$ DEPC water. The sequences for all primers are as follows: GAPDH forward primer, 5'-ACCCAGAAGACTGTGGATGG-3'; GAPDH reverse primer,5'-TTCTAGACGGCAGGTCAGGT-3'; RIPK1 forward primer, 5' TGCACAGCAAAGACCTTACG-3'; RIPK1 reverse primer, 5'-TTGTTCCAAAGCCATGTGAG-3'; RIPK3 forward primer, 5'-CAAGGAGGGACAGAAATGGA-3'; RIPK3 reverse
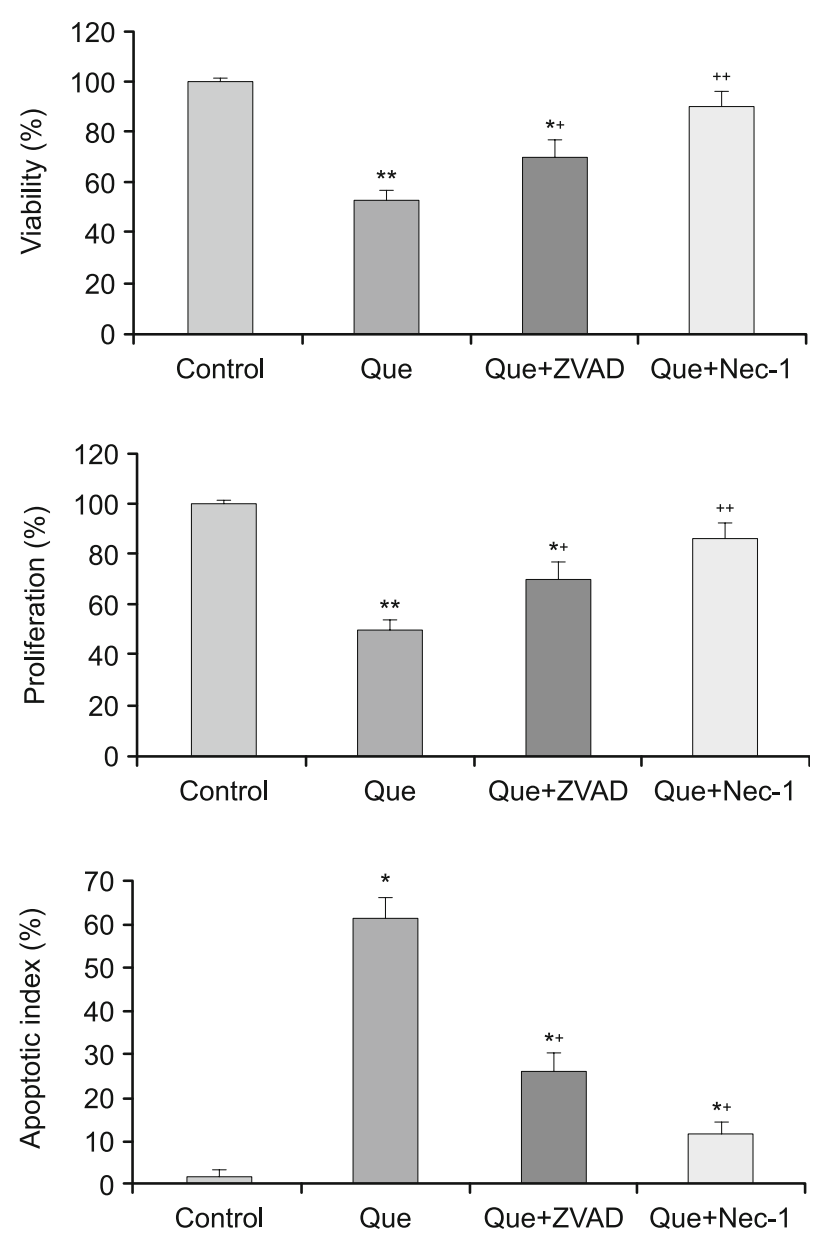

Fig. 1. Viability and proliferation of control and experimental groups. Values are expressed as mean \pm SD. ${ }^{*} p<0.01,{ }^{* *} p<0.001,{ }^{\dagger} p<0.01$, ${ }^{+} \mathrm{p}<0.001$; * and ${ }^{\dagger}$ symbols respectively indicate comparison to control and Que-treated cells. 
primer, 5'-TTGTGGAACCTGCTCCTCTT-3'; Bax forward primer, 5'- GCTGGACATTGGACTTCCTC-3'; Bax reverse primer, 5'- ACCACTGTGACCTGCTCCA-3'; Bcl-2 forward primer, 5'- GCTGGACATTGGACTTCCTC-3'; Bcl-2 reverse primer, 5'-GCTGGACATTGGACTTCCTC-3'; Caspase-3 forward primer, 5'-GAGGCCGACTTCTTGTATGC-3'; caspase-3 reverse primer, 5'- CGGTTAACCCGGGTAAGAAT -3'. PCR amplification was done in 40 cycles using the following program: $95^{\circ} \mathrm{C}$ for $10 \mathrm{~min}-$ utes, $95^{\circ} \mathrm{C}$ for $15 \mathrm{~s}, 5^{\circ} \mathrm{C}$ for $30 \mathrm{~s}$ and $60^{\circ} \mathrm{C}$ for $34 \mathrm{~s}$. Data were analyzed using the $2^{-\Delta \Delta \mathrm{CT}}$ method. Expression values were corrected for the housekeeping gene GAPDH (12).

\section{Statistical analysis}

The data were compared by one-way analysis of variance (ANOVA). This was followed by post hoc pair-wise comparison using the Bonferroni t-procedure. Statistical analysis was performed using the SPSS for Windows, Version 15.0. $\mathrm{p}<0.05$ was considered as significant.

\section{Results}

\section{Cell viability and proliferation}

Cell viability in the Que treated cells was significantly decreased compared to control untreated cells $(p<0.01)$. In Que + ZVAD-treated cells, viability was significantly increased compared to absence of ZVAD. In Que + Nec-1 treated cells, viability was significantly increased compared to absence of Nec-1 $(p<0.05)$. These results are shown in Figure 1. The proliferation of MCF-7 cells was significantly decreased by Que treatment $(\mathrm{p}<0.01)$. Que + ZVAD induced a significant increase in proliferation of MCF-7 cells compared to absence of ZVAD ( $p<0.01)$. Que plus Nec-1 caused a significant increase in proliferation of MCF-7 cells compared to absence of Nec-1 (Figs 1 and 2). Viability and proliferation of MCF-10a cells was not changed in response to Que (the results are not shown).

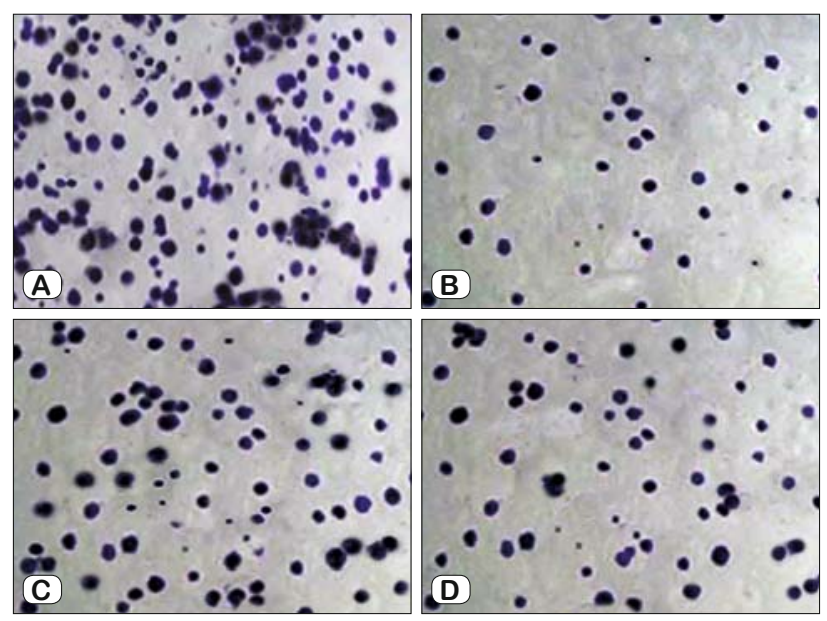

Fig. 2. Colonogenicity assay in control and experimental groups. A, control untreated cells; B, Que-treated cells; C, Que+ZVAD-treated cells; D, Que+Nec-1- treated cells; All magnifications are x400.
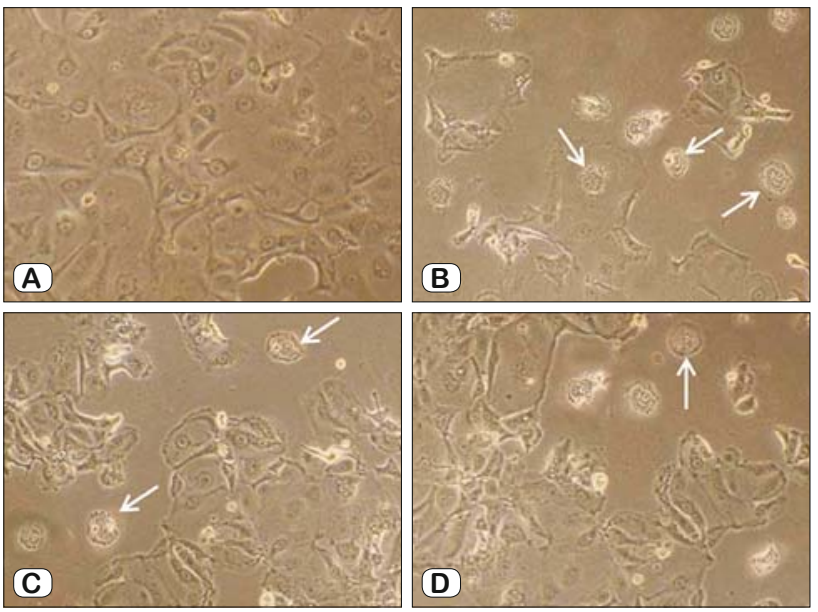

Fig. 3. Morphology of MCF-7 cells in various groups. A, control untreated cells; B, Que-treated cells; C, Que+ZVAD-treated cells; D, Que+Nec-1- treated cells; arrows indicate apoptosis, all magnifications are $\mathrm{x} 400$.
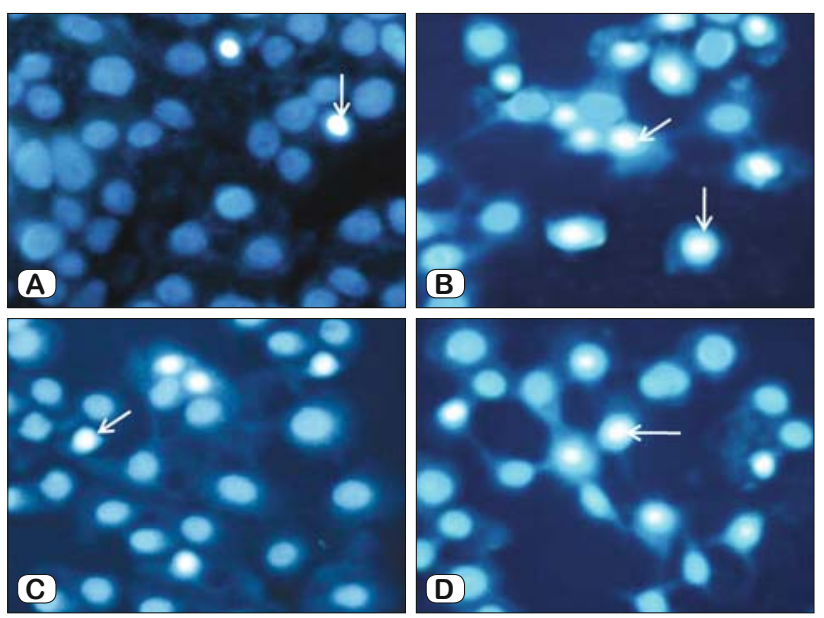

Fig. 4. DAPI staining of MCF-7 cells in various groups. A, control untreated cells; B, Que-treated cells; C, Que+ZVAD- treated cells; D, Que+Nec-1- treated cells; arrows indicate apoptosis, all magnifcations are $\mathrm{x} 400$.

\section{Morphology evaluation}

In control group a few number of MCF-7 cells had round morphology. In Que treated cells a large number of cells showed apoptotic morphology including round shape, cell membrane blebbing and nucleus condensation. The number of apoptotic cells in presence of ZVAD was increased compared to absence of it. In Que plus Nec-1 treated cells the number of MCF-7 cells with apoptosis morphology was decreased in comparison to absence of Nec-1 (Fig. 3). Morphology of MCF-10a cells was not changed in response to Que (the results are not shown).

\section{DAPI staining}

Que-treated MCF-7 cells exhibited a large number nuclei with chromatin condensation which indicates cell apoptosis. Apoptotic 
index in Que-treated cells was significantly increased $(\mathrm{p}<0.001)$. In Que plus ZVAD-treated cells apoptotic index was significantly decreased in comparison to absence of ZVAD. In Que plus Nec1 apoptotic index was significantly increased in comparison to absence of Nec-1. These results are reported in Figures 4 and 5. Apoptotic index in MCF-10a cells was not changed in response to Que (the results are not shown).

\section{Real time}

In Que-treated cells Bax expression was significantly increased while expression of Bcl-2 was significantly decreased in comparison to control untreated cells. Treatment of Que $+\mathrm{Nec}-1$ induced a significant decrease in Bax expression while, Bcl-2 was significantly increased in comparison to control cells. Treatment with Que + ZVAD could not significantly change the expression of Bax and Bcl-2 genes. Expression of Caspase-3 was significant-

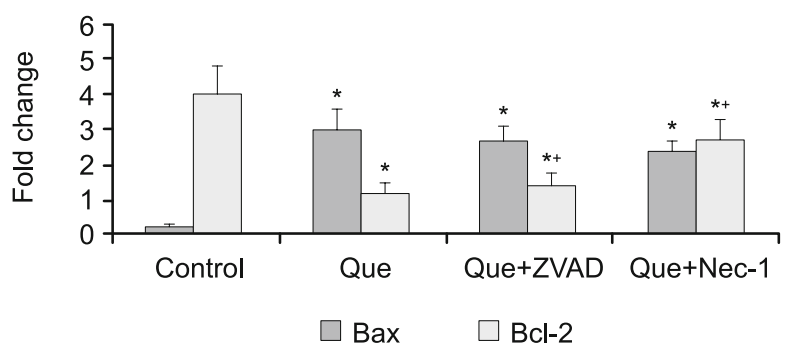

Fig. 5. Apoptotic index of control and experimental groups. Values are expressed as mean $\pm \mathrm{SD} .{ }^{*} \mathbf{p}<\mathbf{0 . 0 0 1},{ }^{\dagger} \mathbf{p}<\mathbf{0 . 0 0 1}{ }^{*}$ and $^{\dagger}$ symbols respectively indicate comparison to control and Que-treated cells.
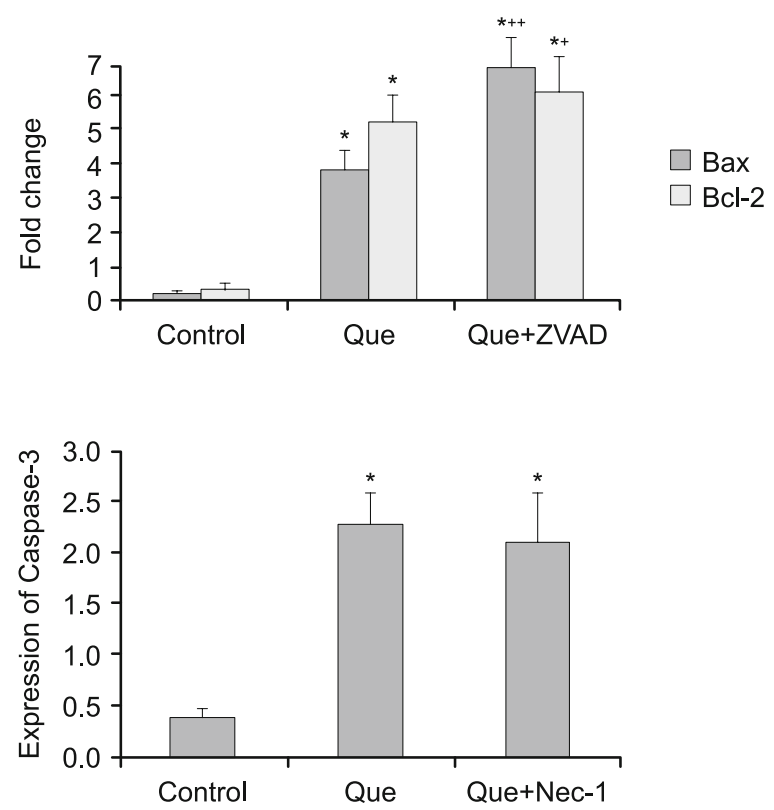

Fig. 6. Gene expression in various groups. Expression normalized to average of housekeeping gene (GAPDH). Values are expressed as mean \pm SD. ${ }^{*} \mathbf{p}<0.001,{ }^{\dagger} \mathbf{p}<0.01,{ }^{\dagger+} \mathbf{p}<0.001 ;{ }^{*}$ and ${ }^{\dagger}$ symbols respectively indicate comparison to control and Que-treated cells. ly increased in Que compared to control untreated cells. ZVAD could completely block the expression of Caspase- 3 in MCF-7 cells. Nec-1 had no effect on expression of Caspase-3. The expression of RIPK1 and RIPK3 genes was significantly increased in Que-treated cells in presence of ZVAD compared to absence of it. Expression of RIPK1 and RIPK3 in presence or absence of ZVAD was significantly increased in Que-treated cells compared to the control (Fig. 6). Expression of RIPK1 and RIPK3 was completely blocked by Nec-1. Gene expression in MCF-10a cells was not significantly changed in response to Que (the results are not shown).

\section{Discussion}

This study demonstrated that Que could effectively suppress MCF-7 cells growth by stimulation of both apoptosis and necroptosis signaling pathways. To determine the role of necroptosis, the pan-caspase inhibitor Z-VAD, which irreversibly binds to the catalytic site of caspases, was used to selectively inhibit the apoptotic pathway. In presence of ZVAD, cell viability was significantly increased in comparison to absence of ZVAD. In the presence of the necroptosis inhibitor Nec-1, the viability of MCF-7 cells was increased compared to absence of Nec-1. In addition, viability percentage of MCF-7 cells in presence of Nec-1 was higher than the presence of ZVAD. Therefore, Nec-1 was slightly more effective than ZVAD in protecting cancer cells.

Using cologenecity assay to measure cell proliferation, we observed that Que significantly inhibited proliferation of MCF7 cells. The inhibitory effect of Que in presence of ZVAD was increased in comparison to presence of Nec-1. These findings indicate that necroptosis plays an important role in inhibition of proliferation and viability of MCF-7 cells. As mentioned in results, the viability and proliferation of MCF-7 cells in absence of ZVAD and Nec-1 were significantly reduced in comparison to presence of them. Hence, apoptosis and necroptosis may have synergetic effects on cell death in MCF-7 cells. On the other hand Que in presence of ZVAD could decrease viability and proliferation of MCF-7 cells compared to untreated control group. Thus, Que may be useful for inducing necroptosis in apoptosis resistant cancers.

To confirm the role of necroptosis, we examined the mRNA expression level of central regulators of necroptosis in MCF-7 cells by real-time. In our study expression of RIPK1 and RIPK3 was low in control untreated cells. Moriwaki et al found that RIPK1 and RIPK3 expression was reduced in primary colon cancer tissues compared with normal adjacent tissues (13). Similar reduction in RIPK3 expression was also reported in patients with acute myeloid leukemia (14). These observations are consistent with the fact that the majority of cancer cell lines lack RIPK3 and RIPK1 expression.

Compared to control untreated cells, the expression of RIPK1 and RIPK3 genes in Que treated cells showed a nearly 19 fold and 17.3 fold increase, respectively. The expressions of RIPK1 and RIPK3 were significantly increased after treatment with Que plus ZVAD in comparison to absence of ZVAD. It is known that 
caspase inhibitors elevate expression of RIPK1 and RIPK3 in cancer cells (7). For example, HT-29 cells under cobalt chloride plus ZVAD treatment expressed increased levels of RIPK1, RIPK3 (15). In addition, expression of these genes was completely blocked by Nec-1 treatment in present study. These results indicate that Que can induce cell death in MCF-7 cells via RIPK1 and RIPK3 dependent necroptosis pathway. When expression of RIPK1 was blocked by Nec-1, viability and proliferation of MCF-7 cells were significantly increased. Hence, there is an inverse relationship between expression of necroptosis regulator genes and survival of Que-treated MCF-7 cells.

To study whether the cytotoxicity of Que in MCF-7 cells was associated with induction of apoptosis, we used the DAPI staining method, morphology assessments and mRNA expression level of Bax, Bcl-2 and Caspase-3. DAPI is a DNA-binding fluorescent dye used to visualize DNA fragmentation. Apoptosis, as detected by DAPI staining, was significantly higher in MCF-7 cells treated with Que than the control. The significant percentage of cell death detected by DAPI staining was consistent with that detected by MTT assay. In addition, morphological observation revealed typical apoptosis characteristics; including cell shrinkage, membrane blebbing, condensed contents, and a rounded body, further demonstrating Que-induced apoptosis of MCF-7 cells. Morphology assessments confirm the results of DAPI staining and are consistent with viability and proliferation percentage in control and experimental groups.

The death mode switch is at least partially due to the conversion from mitochondrial inner membrane permeability to mitochondrial outer membrane permeability, which is associated with Bax translocation (16). In this study, Que could enhance Bax expression nearly 15 fold and reduce expression of Bcl-2 nearly 3.33 fold in comparison to the control. These data confirm the results obtained by DAPI staining which revealed the Que could effectively induce apoptosis in MCF-7 cells. Bax expression is closely related to development of various malignant tumors and is significantly reduced in some of them (17). In our study, we found the expression of Bax was significantly decreased after treatment with Que plus Nec-1 in comparison to absence of Nec-1. Also, we demonstrated that in presence of ZVAD the expression of Bax and Bcl-2 genes was not significantly changed compared to absence of ZVAD. While in presence of $\mathrm{Nec}-1$ the expression of $\mathrm{Bcl}-2$ was increased in comparison to absence of Nec-1. Hence, Nec-1 may suppress apoptosis in Que -treated cells. Interestingly, in presence of Nec-1 the expression of Caspase-3 was not changed. Therefore, Nec-1 may inactivate other caspases or inactivate caspase-independent apoptotic signaling pathways. As shown in results the expression of Caspase-3 was completely inhibited by ZVAD. Interestingly, Que in presence of ZVAD also induced apoptosis.

Many of the apoptotic features observed are not regulated by caspase activation. Such features include cytoplasmic and nuclear shrinkage, mitochondrial depolarisation, peripheral chromatin condensation and, in some instances, oligonucleosomal DNA fragmentation. Furthermore, there are now several examples of cells surviving the activation of caspases, suggesting that caspases alone are not sufficient for the induction of apoptosis (18).
Our results showed that necroptosis may possess more effects in killing cells in comparison to apoptosis. However, apoptosis and necroptosis are not completely distinct events. For example, overexpression of Bcl-2 was found sufficient to prevent the necrotic death of neuronal and non-neuronal cells $(19,20)$. It has also been reported that RIPK1 can regulate not only necroptotic but also apoptotic cell death (21).

In summary, this study demonstrated that Que at the dose of $50 \mu \mathrm{M} / \mathrm{ml}$ could effectively suppress viability and proliferation of MCF-7 cells by activation of both apoptosis and necroptosis signaling pathways. Apoptosis was induced via increasing expression of Bax and Caspase- 3 and decreasing expression of Bcl-2 genes. Necroptosis was induced by increasing expression of RIPK1 and RIPK3. In our knowledge, the present study is the first demonstration of Que-induced non-apoptotic necroptosis in MCF-7 cells and provides potential strategies for developing agents against apoptosis-resistant cancers. Future studies are needed to increase our knowledge about cell death signaling pathways in Que-treated cancer cells.

\section{References}

1. Tinoco G, Warsch S, Glück S, Avancha K, Montero AJ. Treating breast cancer in the 21 st century: emerging biological therapies. J Cancer 2013; 4 (2): 117-132.

2. Gadhwal MK, Patil S, D’Mello P et al. Synthesis, characterisation and antitumour activity of some quercetin analogues. Indian J Pharm Sci 2013; 75 (2): 233-237.

3. Gibellini L, Pinti M, Nasi M et al. Quercetin and Cancer Chemoprevention. Evid Based Complement Alternat Med 2011; 2011: 591356.

4. Meng MB, Wang HH, Cui YL et al. Necroptosis in tumorigenesis, activation of anti-tumorimmunity, and cancertherapy. Oncotarget 2016. In Press.

5. Eisenberg-Lerner A, Bialik S, Simon HU, Kimchi A. Life and death partners: apoptosis, autophagy and the cross-talk between them. Cell Death Differ 2009; 16 (7): 966-975.

6. Cory S, Adams JM. The Bcl 2 family: regulators of the cellular lifeor-death switch. Nat Rev Cancer 2002; 2 (9): 647-656.

7. Li J, McQuade T, Siemer AB et al. The RIP1/RIP3 necrosome forms a functional amyloid signaling complex required for programmed necrosis. Cell 2012; 150 (2): 339-350.

8. Cho Y, Challa S, Moquin D et al. Phosphorylation-driven assembly of the RIP1-RIP3 complex regulates programmed necrosis and virus-induced inflammation. Cell 2009; 137 (6): 1112-1123.

9. Orazizadeh M, Khodadadi A, Bayati V et al. In Vitro toxic effects of Zinc oxide nanoparticles on rat adipose tissue-derived mesenchymal stem cells. Cell J 2015; 17 (3): 412-421.

10. Zhou C, Zhong Q, Rhodes LV et al. Proteomic analysis of acquired tamoxifen resistance in MCF-7 cells reveals expression signatures associated with enhanced migration. Breast Cancer Res 2012; 14 (2): R45.

11. Orazizadeh M, Daneshi E, Hashemitmar M, Absalan F, Khorsandi L. Protective effect of beta-carotene against titanium dioxidenanoparticles induced apoptosis in mouse testicular tissue. Andrologia 2015; 47 (7): 816-825. 


\section{$123-128$}

12. Khorsandi L, Nejad-Dehbashi F, Ahangarpour A, Hashemitabar M. Three-dimensionaldifferentiationof bone marrow-derivedmesenchymalstemcellsintoinsulin-producingcells. TissueCell 2015; 47 (1): 66-72.

13. Moriwaki K, Bertin J, Gough PJ, Orlowski GM, Chan FK. Differential roles of RIPK1 and RIPK3 in TNF-induced necroptosis and chemotherapeutic agent-induced cell death. Cell Death Dis 2015; 6: e1636.

14. Nugues AL, El Bouazzati H, Hetuin D et al. RIP3 is downregulated in human myeloid leukemia cells and modulates apoptosis and caspasemediated p65/RelA cleavage. Cell Death Dis 2014; 5: e1384.

15. Wang HY, Zhang B. Cobalt chloride induces necroptosis in human colon cancer HT-29 cells. Asian Pac J Cancer Prev 2015; 16 (6): 2569-2574.

16. Han W, Xie J, Li J, Liu Z, Hu X. Necrostatin-1 reverts shikonininduced necroptosis to apoptosis. Apoptosis 2009; 14 (5): 674-686.
17. Han W, Xie J, Fang Y, Wang Z, Pan H. Nec-1 enhances shikonininduced apoptosis in leukemia cells by inhibition of RIP-1 and ERK1/2. Int J Mol Sci 2012; 13 (6): 7212-7225.

18. Donovan M, Cotter TG. Control of mitochondrial integrity by Bcl2 family members and caspase-independent cell death. Biochim Biophys Acta 2004; 1644 (2-3): 133-147.

19. Kane DJ, Ord T, Anton R, Bredesen DE. Expression of bcl-2 inhibits necrotic neural cell death. J Neurosci Res 1995; 40 (2): 269-275.

20. Shimizu S, Eguchi Y, Kosaka H et al. Prevention of hypoxia-induced cell death by Bcl-2 and Bcl-xL. Nature 1995; 374 (6525): 811-813.

21. Vandenabeele P, Grootjans S, Callewaert N, Takahashi N. Necrostatin-1 blocks both RIPK1 and IDO: consequences for the study of cell death in experimental disease models. Cell Death Differ 2013; 20 (2): $185-187$. 